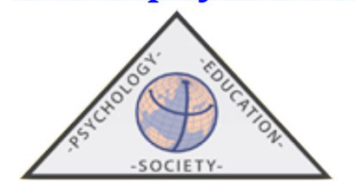

\title{
Estudiar en prisión: la situación actual de las mujeres en contextos penitenciarios españoles
}

\author{
Fanny T. AÑÃNOS BEDRIÑANA ${ }^{1}$, Fernando GIL CANTERO ${ }^{2}$, Rocío RAYA MIRANDA ${ }^{1}$ \\ y Fátima SOTO NAVARRO ${ }^{1}$ \\ ${ }^{1}$ Universidad de Granada \\ ${ }^{2}$ Universidad Complutense de Madrid
}

(Recibido el 12 de Mayo de 2020, Aceptado el 24 de Noviembre de 2020)

RESUMEN: En este trabajo se presentan los resultados obtenidos en una investigación realizada en 31 entidades penitenciarias españolas y, tiene por objeto analizar el acceso de las mujeres penadas a los distintos niveles educativos, especialmente en los estudios medios o superiores, y su relación con distintos factores de riesgo y protección intervinientes. La muestra global (310 mujeres, 30,1\%) se ha seleccionado utilizando muestreo bietápico, de la población de mujeres en cumplimiento de condena en medio abierto. La metodología que se ha usado combina métodos cuantitativos y cualitativos, aplicados a los instrumentos desarrollados diseñados ad hoc (cuestionario de mujeres y entrevistas personales, y cuestionario de profesionales). Los resultados muestran que la mayor parte abandonan los estudios en las enseñanzas básicas, observándose distintos factores de riesgo; sin embargo, las pocas que continúan o retoman los estudios medios o superiores en prisión, lo consideran como un factor de protección frente al ambiente penitenciario, permitiéndoles gestionar de forma inteligente y saludable el tiempo en contextos penitenciarios, mejorando las relaciones entre las presas, con las familias y los trabajadores de prisión. También consideran que les puede resultar útil para el futuro, especialmente, en el ámbito laboral.

Palabras clave: educación, prisión, formación profesional, estudios superiores, factor de protección.

\section{Study in Prison: The Current Situation of Women in the Spanish Penitentiary Context}

\begin{abstract}
This aim of this study was to feature the results obtained on a social research, done in 31 correctional jurisdictions in Spain, and to assess the access to the different education levels by women prisoners, especially in the higher education cases and their relation to the different risk factors. The participants ( $\mathrm{n}=310$ women sentenced in open prisons, 30,1\%) were selected in a two-stage sampling. The methodology used, combined a quantitative and qualitative methods (women's questionnaire, personal interviews and professionals questionnaire). The results showed that the majority of participants left the school in an early stage, due to the risk factors. However, the participants who continued or returned to the education system in prison, considered it as a protection factor in this difficult environment, allowing them to manage and control their time in prison in a safer and healthier way, improving the relationships
\end{abstract}


with the rest of the prisoners, their families and the prison officers. In addition, the participants considered that studying in prison could be useful and beneficial for their professional career.

Keywords: education, prison, vocational training, higher education, protection factor.

Correspondencia: Fanny T. Añaños. Campus Universitario de Cartuja, 18071 Granada. E-mail: fanntab@ugr.es

\section{Introducción}

Como es sabido, las reglas mínimas de las Naciones Unidas para el tratamiento de los reclusos (Council of Europe, 2006, p.4) destacan que, para favorecer la reinserción, las autoridades penitenciarias "deberán ofrecer educación, formación profesional y trabajo." Por esto, una de las áreas de intervención socioeducativa más interesante en la actualidad dentro del ámbito penitenciario es la referida a la participación de los/as internos/as en los diferentes cursos de educación reglada.

En este artículo tiene como objeto ofrecer a la comunidad científica el análisis de una investigación centrada en recabar, pormenorizadamente, el estado actual en que se encuentran las internas que participan o han participado, antes y durante su estancia en prisión, en el sistema educativo, especialmente en los niveles medio y superior.

La mayoría de los estudios e informes europeos apoyan las propuestas de promoción de la asistencia a cursos de educación formal en las instituciones penitenciarias (Callejo y Viedma, 2008; Council of Europe, 2009) al considerar que es un factor de protección. Esta propuesta tiene su respaldo empírico en que las carreras delictivas más largas y los ingresos penitenciarios más frecuentes correlacionan con los que abandonaron la escuela de forma más temprana (Hawley, Murphy y Souto-Otero, 2013, p.12 y ss.). El Proyecto EURODESIP analiza, específicamente, tanto las dificultades que tienen los internos por su historial previo de aprendizaje como las especiales peculiaridades -limitaciones- de la enseñanza en prisión (Gutiérrez Brito, Viedma Rojas y Callejo Gallego, 2010; Pastore, 2018; Cerezo y Izco, 2019). Como es habitual, la expresión factores de protección o de riesgo a lo largo del artículo indica los elementos personales, sociales u organizativos, externos e internos a los sujetos y a la propia prisión, que pueden favorecer, respectivamente, alejarse de la carrera delictiva o mantenerse en ella.

Otro ámbito académico internacional, especialmente significativo en este tema, es el latinoamericano (Pérez, 2019; Murillo, 2019; Schneider, 2018; Larrea Álvarez, 2014; Sáez, Domínguez y Mendoza, 2014; Scarfó y Zapata, 2013; López, 2010), centrado también en señalar las dificultades, aunque con dos enfoques singulares: uno, que vincula la enseñanza reglada con los derechos humanos y, otro, que cuestiona críticamente el que se reduzca la perspectiva educativa de la reinserción a la asistencia a cursos de educación formal.

Un tema que está adquiriendo una amplia resonancia internacional es el referido a las posibilidades técnicas de conexión vía web con el exterior para favorecer los medios de aprendizaje (Diana, 2013; Makoe, 2016; Moreira, Reis-Monteiro y Machado, 2017; Solé Blanch, 2020). Otro tema que considerar son los peculiares efectos en la identidad profesional de los profesores y tutores que ayudan pedagógicamente a este tipo de estudiantes dadas las especiales circunstancias en las que desarrollan su trabajo (Sumba, Cueva y López, 2019; Vila, 2019).

La mayoría de las investigaciones sobre la educación media y universitaria en prisión en el ámbito internacional destacan unas carencias comunes: imposibilidad de acceder a la web; poco 
material de apoyo o muy antiguo; escaso profesorado y sin preparación pedagógica específica para adaptarse a las situaciones penitenciarias; minusvaloración de estas actividades por parte de los responsables de la prisión; carencia de espacios físicos específicos y acceso limitado a la información (Gutiérrez, Callejo, y Viedma, 2010; López, 2010; Rivera, 2016; UNESCO, 2008).

Del mismo modo, hay una elevada coincidencia cuando se trata de destacar los aspectos positivos: mejora de la convivencia; mejores expectativas de futuro laboral; disminución de los conflictos y mayor colaboración en tareas comunes (Earle, 2011; Maculan, Ronco y Vianello, 2013; Parchuc, 2015; Farley y Pike, 2016).

Finalmente, se considera importante mostrarlos siguientes datos (Tabla $\left.N^{o} 1\right)$ sobre la evolución de las mujeres que participaron en el curso escolar correspondiente en cada uno de los niveles educativos durante los últimos seis períodos publicados por la Secretaría General de Instituciones Penitenciarias (SGIP). Entre los cursos 2012/2013 hasta el 2017/2018 el número de mujeres que cursaba cada nivel se ha mantenido prácticamente constante, concretamente, con respecto a las que cursaban bachillerato o bien FP o Escuela Oficial de Idiomas (en adelante, EOI), se produce un ligero incremento de un 1\%. En cuanto a los estudios universitarios, si exceptuamos el primer curso (2012/2013) que es el que tiene el valor más alto, con un 6\%, de las mujeres realizando dichos estudios, también se produce un incremento en los últimos años situando el porcentaje en un 5,3\% (90 mujeres). Situación que es, en parte, distinta en las enseñanzas básicas, observándose en los dos últimos cursos un ligero descenso en las mujeres (entre 5-6 puntos) en la educación primaria, si bien en esta etapa se sitúa la mayor cantidad de población $(59,3 \%$ en el curso $2017 / 2018)$ y, en la educación secundaria las proporciones suelen mantenerse (22\% el curso 2017-2018).

Tabla 1. Evolución del número de mujeres que cursan estudios de Bachillerato y Universidad

\begin{tabular}{|c|c|c|c|c|c|c|c|c|c|c|c|c|c|c|c|c|c|c|c|c|c|c|c|c|}
\hline & \multicolumn{4}{|c|}{ Curso2012/13 } & \multicolumn{4}{|c|}{ Curso2013/14 } & \multicolumn{4}{|c|}{ Curso2014/15 } & \multicolumn{4}{|c|}{ Curso2015/16 } & \multicolumn{4}{|c|}{ Curso2016/17 } & \multicolumn{4}{|c|}{ Curso2017/18 } \\
\hline & \multicolumn{2}{|c|}{ Hombres } & \multicolumn{2}{|c|}{ Mujeres } & \multicolumn{2}{|c|}{ Hombres } & \multicolumn{2}{|c|}{ Mujeres } & \multicolumn{2}{|c|}{ Hombres } & \multicolumn{2}{|c|}{ Mujeres } & \multicolumn{2}{|c|}{ Hombres } & \multicolumn{2}{|c|}{ Mujeres } & \multicolumn{2}{|c|}{ Hombres } & \multicolumn{2}{|c|}{ Mujeres } & \multicolumn{2}{|c|}{ Hombres } & \multicolumn{2}{|c|}{ Mujeres } \\
\hline & $\mathbf{N}^{\circ}$ & $\%$ & $\mathbf{N}^{o}$ & $\%$ & $\mathbf{N}^{\circ}$ & $\%$ & $\mathbf{N}^{0}$ & $\%$ & $\mathbf{N}^{0}$ & $\%$ & $\mathbf{N}^{\circ}$ & $\%$ & $\mathbf{N}^{\circ}$ & $\%$ & $\mathbf{N}^{o}$ & $\%$ & $\mathbf{N}^{0}$ & $\%$ & $\mathbf{N}^{\circ}$ & $\%$ & $\mathbf{N}^{\circ}$ & $\%$ & $\mathbf{N}^{\circ}$ & $\%$ \\
\hline Ed. Básica & 10863 & 64,2 & 1180 & 61,2 & 10160 & 61,6 & 1158 & 61,6 & 9891 & 61,7 & 1125 & 60,1 & 9198 & 59,3 & 1185 & 64,3 & 8734 & 57,4 & 1014 & 58,4 & 8619 & 57 & 1006 & 59,3 \\
\hline Ed. Secundaria & 3541 & 20,9 & 405 & 21 & 3658 & 22,2 & 435 & 23,1 & 3426 & 21,4 & 443 & 23,7 & 3418 & 22 & 362 & 19,7 & 3511 & 23,1 & 376 & 21,7 & 3592 & 23,7 & 372 & 22 \\
\hline Bach., FP, EOI & 453 & 2,7 & 63 & 3,3 & 548 & 3,3 & 64 & 3,4 & 546 & 3,4 & 66 & 3,5 & 662 & 4,3 & 78 & 4,3 & 675 & 4,4 & 77 & 4,4 & 559 & 3,7 & 74 & 4,4 \\
\hline Universitarias & 1123 & 6,6 & 116 & 6 & 979 & 5,9 & 63 & 3,3 & 940 & 5,9 & 80 & 4,3 & 1003 & 6,5 & 75 & 4 & 1058 & 7 & 88 & 5,1 & 1078 & 7,1 & 90 & 5,3 \\
\hline Otras enseñanzas & 949 & 5,6 & 163 & 8,5 & 1159 & 7 & 161 & 8,6 & 1203 & 7,6 & 157 & 8,4 & 1235 & 7,8 & 141 & 7,7 & 1227 & 8 & 181 & 10,4 & 1283 & 8,5 & 152 & 9 \\
\hline Total & 16929 & 100 & 1927 & 100 & 16504 & 100 & 1881 & 100 & 16006 & 100 & 1871 & 100 & 15516 & 100 & 1841 & 100 & 15205 & 100 & 1736 & 100 & 15131 & 100 & 1694 & 100 \\
\hline
\end{tabular}

Fuente: Elaboración propia a partir de la Secretaría General de Instituciones Penitenciarias (SGIP, 2013, 2014, 2015, 2016, 2017, 2018).

\section{Métodos}

La presente investigación se enmarca en el Proyecto $\mathrm{I}+\mathrm{D}+\mathrm{i}$ "Procesos de reinserción socioeducativa y acompañamiento a reclusas en semilibertad" -REINAC- (Ref.EDU2016-79322-R), financiado por el Ministerio de Economía y Competitividad, la Agencia Estatal de Investigación y FEDER, del gobierno español, liderado por la Universidad de Granada. El trabajo ha sido 
avalado y aprobado por la Secretaría General de Instituciones Penitenciarias y la Consejería de Justicia de la Generalitat de Cataluña, llegando a investigar 31 centros.

\section{Método, participantes y diseño muestral}

La investigación ha empleado métodos cualitativos y cuantitativos, de forma complementaria, aplicados a los cuestionarios mixtos y entrevistas, diseñados ad hoc. La población participante son todas las mujeres que se encuentran en tercer grado o segundo grado especial en proceso de semilibertad, en el medio abierto del sistema penitenciario español. Estos grados penitenciarios, que no jurídicos, establecen la posibilidad de un estilo de vida y un programa de intervención específico de tránsito a la libertad, tanto dentro como fuera del establecimiento penitenciario, según cada caso. La muestra general está compuesta por 310 mujeres reclusas (30,1\% de la población total), obtenida en 31 establecimientos penitenciarios en medio abierto, distribuidos en 13 Comunidades Autónomas de las 17 existentes. Adicionalmente se ha obtenido otro punto de vista a través de un cuestionario específico para los profesionales. En total, este cuestionario lo han realizado 66 profesionales de 18 centros distintos.

Para la obtención de la muestra se siguió un muestreo bietápico. En la primera etapa, el criterio fue el de representación territorial y de mayor ratio de mujeres; en la segunda etapa, una vez situados en los centros seleccionados, el criterio fue al azar entre las mujeres que querían participar y tras firmar el consentimiento voluntario $\mathrm{y}$, teniendo como criterio de inclusión el paso previo en régimen ordinario de prisión. El protocolo para los cuestionarios de profesionales fue diferente ya que se enviaron por correo postal a todos los centros seleccionados antes de que acudieran los encuestadores; en algunos casos se recogieron al terminar la visita, mientras que en otros remitieron posteriormente por correo postal. El margen de error de la muestra (con un nivel de confianza del 95\%) es de 4.5 puntos. El periodo de trabajo de campo abarcó desde junio de 2018 a marzo de 2019.

El cuestionario de mujeres consta de 115 ítems dividido en 6 bloques temáticos, de las que se han analizado algunas preguntas de los bloques I, II y III, cuyos resultados están en las Tablas 2, 3 y 4. El cuestionario de profesionales consta de 100 ítems, igualmente dividido en 6 bloques, de las que se extrajo las preguntas relacionadas con las analizadas en el cuestionario de mujeres.

Los análisis corresponden a estudios descriptivos básicos realizados usando SPSS V.24 y análisis de contenido de las respuestas abiertas y de las entrevistas.

\section{Resultados}

\section{Los estudios. La voz de las de las mujeres}

En la Tabla $N^{\circ} 2$ se observa que, de un total de 310 mujeres, 103 (33,3\%) cursaron estudios medios y superiores antes de ingresar en prisión, frente al 66,2\% que sólo realizaron estudios básicos o abandonaron los estudios en los niveles obligatorios. Así, un 9,7\% (30) afirma haber realizado estudios de FP, aunque de esas un 4,2\% (13) no ha completado dichos estudios. Un 12,6\% (39) afirma haber realizado estudios de bachillerato o de COU antes de ingresar en prisión. Si bien dentro de ese porcentaje 4,8\% (15) reconoce que es incompleta. Respecto a los estu- 
dios superiores universitarios, lo han realizado un $11 \%$ (34) y un 2,6\% (8) de forma incompleta. De las carreras realizadas las más numerosas con una frecuencia de 4 son Ciencias empresariales-económicas y Derecho, seguida con 3 en Enfermería, luego 2 en Relaciones públicas y, 1 en Informática, Psicología, Periodismo, Arquitectura, Humanidades, Marketing y Trabajo social, respectivamente. En total tenemos 103 (33,2\%) mujeres que reconocen haber estudiado o bien hasta los 18 años o bien hasta los 24 años antes de ingresar en prisión.

Tabla 2. Nivel de estudios antes de entrar a prisión

\begin{tabular}{lccc}
\hline & Completados & No completados & Total \\
\hline Sin estudios & - & $34(16,6 \%)$ & $34(11 \%)$ \\
Primaria & $44(14,2 \%)$ & $59(19 \%)$ & $103(33,2 \%)$ \\
Secundaria (ESO/BUP) & $27(8,7 \%)$ & $41(13,2 \%)$ & $68(22 \%)$ \\
Formación Profesional (FP) oficial no universitaria & $17(5,5 \%)$ & $13(4,2 \%)$ & $30(9,7 \%)$ \\
Estudios de bachillerato, COU, estudios preuniversitarios & $24(7,7 \%)$ & $15(4,8 \%)$ & $39(12,6 \%)$ \\
Estudios universitarios & $26(8,4 \%)$ & $8(2,6 \%)$ & $34(11 \%)$ \\
En blanco & - & - & $2(0,6 \%)$ \\
Total & $138(44,5 \%)$ & $170(54,9 \%)$ & 310 \\
\hline
\end{tabular}

Fuente: Elaboración propia a partir del proyecto REINAC Ref. EDU2016-79322-R.

En cuanto a la educación escolar recibida dentro de prisión, se observa que se han beneficiado 177 (57.1\%) mujeres, entre todos los niveles educativos y, específicamente, 38 (12.3\%) referidas a Bachillerato, EOI y a los estudios superiores universitarios (Tabla $N^{o} 3$ ). Actualmente, se encuentran recibiendo educación escolar 49 (15,8\%), siendo 13 (4,2\%) correspondientes a los estudios de Bachillerato, EOI y estudios superiores.

Tabla 3. Nivel Educativo Cursado en Prisión y En La Actualidad

\begin{tabular}{lcccccc}
\hline & \multicolumn{9}{c}{ Estudios en prisión } & Estudio & Útil \\
\cline { 2 - 6 } & Completados & $\begin{array}{c}\text { No } \\
\text { completados }\end{array}$ & Total & Útil & & \\
\hline Primaria & 25 & 70 & $95(30,6 \%)$ & $90(94,7 \%)$ & $26(8,4 \%)$ & $16(61,5 \%)$ \\
Secundaria & 20 & 24 & $44(14,2 \%)$ & $27(61,4 \%)$ & $10(3,2 \%)$ & $1(10 \%)$ \\
Bachillerato, EOI & 6 & 21 & $27(8,7 \%)$ & $23(85,2 \%)$ & $7(2,3 \%)$ & $5(71,4 \%)$ \\
Estudios universitarios & 4 & 7 & $11(3,5 \%)$ & $7(63,6 \%)$ & $6(1.9 \%)$ & $6(100 \%)$ \\
\hline
\end{tabular}

Fuente: Elaboración propia a partir del proyecto REINAC Ref. EDU2016-79322-R.

De la Tabla $N^{o} 3$ se destaca, en primer lugar, durante su estancia en prisión: un 8,7\% (27) afirma que ha realizado estudios de Bachillerato, EOI, o estudios preuniversitarios, si bien un $6,8 \%$ (21) no los ha completado. Puede ser interesante también considerar que un 85,2\% a la pregunta de si les ha parecido útil realizar estos estudios de bachillerato dentro de la prisión consideran que sí. 
La investigación ha analizado los estudios cursados en prisión, teniendo en cuenta que, por un lado, las estancias en prisión rondan como media en España, en torno a los 9 años, tiempo suficiente para finalizar estos grados de enseñanza, y, por otro, es interesante conocer las percepciones sobre la utilidad de estos estudios. En según lugar, la situación frente a los estudios en la actualidad, según los datos recabados, un 2,3\% (7) reconocen estar cursando el bachillerato o EOI y $71,4 \%$ valoran como útil realizar estos estudios.

Respecto a los estudios superiores se distingue que un 3,5\% (11) dice haber cursado dentro de prisión, un 2,3\% (7) de forma incompleta y que 63,6\% considera útil. Las carreras seguidas refieren a: 3 Derecho, 3 Formación profesional de grado superior, 3 preparación al acceso a la universidad y 2 han homologado un título extranjero. En total tenemos 38 mujeres que reconocen haber estudiado o bien bachillerato, EOI o bien estudios superiores. En cuanto, en este caso, a la pregunta de si se encuentran estudiando ahora, hallamos que un 1,9\% (6) afirma que sí y que el $100 \%$ consideran útil haberse matriculado en estos grados. De las respuestas obtenidas referidas a la elección concreta de estudios superiores, nos encontramos que 2 están cursando Derecho, 1 Formación profesional y otro más acceso a la universidad.

Por su parte, se ha recabado también información mediante entrevistas, acerca de si consideran que "estos estudios ayudan a la vida en libertad y por qué" (por "estos estudios" nos estamos refiriendo a todos los niveles, obligatorios o no, del sistema educativo formal). Pues bien, el dato más alto, $21,3 \%$ (66), corresponde a la valoración de "bastante" que pasa a explicarse por parte de las internas con las categorías, de mayor a menor porcentaje, de: "hay aprendizaje" $(12,9 \%)$, "hay mejora personal" (11\%), "hay expectativas laborales" (7,4\%). Es interesante saber que hay consideraciones negativas al pensar que no les aporta "nada", concretamente: "tienen poca utilidad" (2,6\%), "no ha aprendido nada" (1,9\%) y "no hay mejora personal" (1,6\%).

Para entender con mayor amplitud las situaciones que rodean a las mujeres y sus realidades asociadas a la educación formal, se analiza en la Tabla $N^{o} 4$ si existen diferentes rasgos con relación a variables sociales, culturales y económicos.

Tabla 4. Nivel educativo y rasgos socioculturales y laborales

\begin{tabular}{lcccc}
\hline & Primaria & Secundaria & $\begin{array}{c}\text { Bachillerato, } \\
\text { EOI, FP }\end{array}$ & $\begin{array}{c}\text { Estudios } \\
\text { Universitarios }\end{array}$ \\
\hline Con pareja estable & $71,1 \%$ & $57,9 \%$ & $54,5 \%$ & $58.3 \%$ \\
Con hijos & $94,4 \%$ & $71,1 \%$ & $86,3 \%$ & $83.3 \%$ \\
Nacionalidad(española) & $90,5 \%$ & $75 \%$ & $48,1 \%$ & $75 \%$ \\
Relación cultura gitana & $68,9 \%$ & $39,5 \%$ & $22,7 \%$ & $16,7 \%$ \\
Trabajo antes & $53,3 \%$ & $68,4 \%$ & $68,2 \%$ & $75 \%$ \\
Trabajo ahora & $24,4 \%$ & $44,7 \%$ & $45,5 \%$ & $50 \%$ \\
Edad media abandono estudios(años) & 10,9 & 16,4 & 21,2 & 19,4 \\
N. ${ }^{\circ}$ medio ingresos & 1,8 & 1,3 & 1,2 & 1,5 \\
Tiempo medio de condena (meses) & 59,7 & 49,8 & 35,2 & 41,7 \\
\hline
\end{tabular}

Fuente: Elaboración propia a partir del proyecto REINAC Ref. EDU2016-79322-R. 
De la Tabla $N^{o} 4$ se desprenden situaciones que diferencian aquellas que abandonaron o sólo cursaron los estudios básicos (primaria-secundaria) frente a las que permanecieron en el sistema hasta la educación superior. Sobre las primeras se observa que pertenecen en gran parte a la cultura gitana, abandonaron los estudios bastante antes de la mayoría de edad, tienen más tiempo de condena, ligeramente más ingresos penitenciarios y, además, las que sólo cursaron la educación primaria tienen más hijos; sin embargo, en éstas se observa mayor estabilidad emocional al contar con parejas estables actuales. En el segundo caso, hay entre un $25 \%$ y $50 \%$ de extranjeras, tienen menos relación con la cultura gitana, menos tiempo de condena y mayor posibilidad de reinserción laboral. Si bien en este último punto, en ambos grupos ha habido un descenso importante del empleo respecto a su condición laboral antes de entrar a prisión, pero en el caso de aquellas con más estudios tienen mayores porcentajes de empleo. Con lo cual el hecho de contar con más educación ofrece mayores factores de protección, y, en consecuencia, mejores posibilidades de reinserción social y laboral.

\section{Los estudios. La voz de los/as trabajadores de la prisión}

De los 65 trabajadores de la prisión encuestados, entre funcionarios y contratados, 21,8\% (31) y un 51,5\% (34) consideran que el nivel de beneficio para las internas de asistir a la enseñanza reglada es "bastante" y "mucho", respectivamente. Las razones apuntadas se decantan: primera, por considerar que esos estudios pueden facilitar la "inserción laboral" $(24,2 \%)$; segunda, el $12,1 \%$ considera que esos estudios suponen un "aprendizaje formativo", lo que también se recoge en cierto modo cuando se indica que pueden favorecer una "mejora personal/social" (9,1\%). Tercera, resulta sorprendente, que se considera escaso el beneficio que puede acarrear para favorecer la "inserción social" $(7,6 \%)$.

\section{Discusión}

Según los datos de nuestra investigación, como se ha visto, el número de mujeres que han cursado estudios bien de FP, bachiller, EOI, o bien universitarios antes del ingreso en prisión (33,2\%), así como las que los realizan estando ya ingresadas (16,5\%) es muy escaso. En otros niveles inferiores de enseñanza el número de mujeres es mayor, más alto en primaria que en secundaria (39\% Primaria y 17,4\% Secundaria). En cualquier caso, cabe destacar que son datos más bajos que los recogidos en los Informes de la SGIP (Tabla $\left.N^{o} 1\right)$. Estos datos contrastan, sin embargo, con las valoraciones positivas manifestadas tanto por las internas como por los trabajadores de la prisión que consideran, por diversas razones, muy útiles la realización de estos estudios.

Algunas variables identificadas en la investigación asocian los bajos niveles educativos cursados tanto en primaria como en secundaria con carreras delictivas más largas y prolijas al coincidir, a su vez, con un incremento del abandono escolar y con un mayor número de hijos, coincidiendo con los planteamientos de Hawley, Murphy y Souto-Otero, 2013. La influencia del entorno familiar y social es también importante (García-Vita, 2017), en los que no se prioriza la escuela, así se puede observar una mayor incidencia en grupos culturales desfavorecidos como el colectivo gitano (De la Rica, Gorjón, Miller y Úbeda, 2019; Cárdenas-Rodríguez, Terrón-Caro y Monreal Gimeno, 2019). Todo ello genera mayores dificultades para adquirir una serie de derechos que deberían estar garantizados a través de la educación. 
Por otra parte, en las mujeres que han realizado o realizan estudios de FP, bachiller, EOI o universitarios destaca, simultáneamente, un perfil característico con tres tendencias, por un lado, de vinculación laboral donde o bien tenían trabajo antes de entrar en prisión $(71,8 \%)$, o bien lo tienen actualmente $(51,3 \%)$; por otro, no tenían en un número importante, como hemos visto, antecedentes penales $(84,6 \%)$ y, por último, han realizado cursos de formación profesional destinados directamente a encontrar un trabajo (67.2\%). Estos datos son consistentes con otras investigaciones en los que se indica que la participación en esos niveles altos de enseñanza depende más de estilos de vida previos bien socializados (Brunton-Smith y Hopkins, 2014). Esto implica, entonces, como se muestra en la Tabla $N^{o} 3$, que la dificultad para retomar los estudios dentro de la prisión depende, a su vez, del momento, más o menos temprano, en el que abandonan los estudios. En cualquier caso, estos datos no deberían enfocarse, en ninguna circunstancia, para minusvalorar la responsabilidad del sistema penitenciario para favorecer la participación de las internas en estos niveles de enseñanza y, por tanto, para beneficiarse de los efectos educativos del estudio (Silva, 2020; Touriñán, 2019; Bárcena, 2019).

Los efectos positivos de la realización de estos estudios en prisión hay cierta consistencia con otras investigaciones (Murillo, 2019; Añaños-Bedriñana, 2013; Gutiérrez, Callejo, y Viedma, 2010) tanto en la valoración global ("bastante positivo") como en las razones concretas ("aprendizaje", "seguridad", "bienestar", "satisfacción personal", "alejamiento físico de las situaciones conflictivas", "menos consumo de drogas", "expectativas laborales"). Coincidimos con Murillo y Añaños-Bedriñana en que esta participación es percibida por las mujeres como "un factor protector", tanto en la actualidad como futuras, especialmente laborales.

\section{Conclusiones}

La realización del presente trabajo investigador supone adentrarnos al contexto punitivo español, que pese a las limitaciones y controles del medio, así como de la enorme burocracia y un delicado y complejo proceso logístico, se ha conseguido analizar una muestra más que significativa de mujeres, a partir de la cual se ofrecen los alcances educativos-sociales y se definen las futuras líneas de investigación.

Así, se evidencia que las mujeres en el medio penitenciario español que han realizado estudios de FP, bachiller, EOI o estudios superiores es menor que los niveles inferiores $(16,5 \%$ vs 56,4\%), como lo es también las que acceden voluntariamente a realizar estos estudios o se incorporan a los mismos tras haberlos abandonado. La baja formación educativa y abandono escolar previo a prisión en los niveles básicos responden a una serie de factores de riesgo y que se relacionan con las carreras delictivas, mayor precariedad y dificultad social, así como la influencia del entorno cultural.

Los estudios universitarios que más han realizado son ciencias empresariales-económicas y derecho, seguidas de enfermería, relaciones públicas, informática, psicología, periodismo arquitectura, humanidades, marketing y trabajo social. En prisión, los grados más elegidos son derecho y formación profesional de grado superior.

La consideración mayoritaria de realizar estos estudios son una utilidad autopercibida a razón de: ayuda para el futuro, para su propio aprendizaje y formación o para gestionar el tiempo penitenciario. Los trabajadores también consideran muy positiva al respecto, relacionándolo a 
un aprendizaje formativo y mejorar su personalidad social; sin embargo, éstos consideran muy escaso el beneficio entre cursar estos estudios y el logro de la inserción social, lo cual llama a replantearse el modelo educativo en este contexto, la proyección sociopersonal y la coherencia a la realidad de estas mujeres.

La participación en los estudios en todos sus niveles, pero especialmente nivel medio y superior, es un claro factor de protección, por lo que exhortamos a las instancias penitenciarias la puesta en marcha de todas las variables que motiven y favorezcan el incremento de internas que continúan sus estudios, haciéndolos, accesibles, asequibles, adaptables y aceptables (ONU, 1966), inclusivos, equitativos y de calidad (UNESCO, 2015), lo cual incidirá en un mayor bienestar y desarrollo personal y en una mejor reinserción-inserción social.

\section{Agradecimientos}

Agradecemos la financiación de este trabajo al Ministerio de Economía, Industria y Competitividad, la Agencia Española de Investigación y FEDER. Agradecemos el aval de la Secretaría General de Instituciones Penitenciarias y la Consejería de Justicia de la Generalitat de Catalunya. Proyecto $\mathrm{I}+\mathrm{D}+\mathrm{i}$ "Procesos de reinserción y acompañamiento a mujeres en semilibertad" (REINAC), Ref. EDU2016-79322-R.

\section{Referencias}

Añaños-Bedriñana, Fanny T. (2013). Formación educativa previa ante las discriminaciones: las mujeres reclusas en España. Revista de Educación, 360, enero-abril, 91-118. DOI:10.4438/1988-592X-RE-2013-360-222

Bárcena, F. (2019). La intimidad del estudio como forma de vida. Teoría de la Educación. Revista Interuniversitaria, 31(2), 41-67. DOI:10.14201/teri.20411

Brunton-Smith, I., and Hopkins, K. (2014). The impact of experience in prison on the employment status of prisoners after release: Findings from the first 3 waves of Surveying Prisoner Crime Reduction (SPCR). Report for Ministry of Justice. United Kingdom. Extraído el 11 de junio de 2020 desde https:/assets.publishing.service.gov.uk/government/uploads/system/ uploads/attachment_data/file/296320/impact-of-experience-in-prison-on-employmentstatus-of-longer-sentenced-prisoners.pdf

Callejo, J. y Viedma Rojas, A. (Eds.) (2008). EURODESIP: Diagnosis of State of Higher Education in Penal Institutions in Europe.

Cárdenas-Rodríguez, R., Terrón-Caro, T. y Monreal Gimeno, M.C. (2019). Educación Primaria y alumnas gitanas. Análisis de las barreras sociales en contextos de exclusión. Revista de Investigación Educativa, 37(1), 75-91. DOI:10.6018/rie.37.1.326221

Cerezo, A. I. y Izco, M. (2019). Young Foreign Women in Prison: A Case Study in Malaga Prison, TEMIDA, 22(3), 235-261. https://doi.org/10.2298/TEM1903235C

Council of Europe (2006). European Prison Rules. Extraído el 4 de abril de 2020 desde https:// rm.coe.int/european-prison-rules-978-92-871-5982-3/16806ab9ae

CouncilofEurope(2009). Councilconclusionsof 12 May2009onaStrategicFrameworkforEuropean Cooperation in Education and Training. Extraído el 4 de abril de 2020 desde https://eurlex.europa.eu/legal-content/EN/TXT/PDF/?uri=CELEX:52009XG0528(01)\&from=EN 
De la Rica, S., Gorjón, L., Miller, L. y Úbeda, P. (2019). Situación de la población gitana en España en relación al empleo y la pobreza. Madrid: ISEAK. Fundación Secretariado Gitano.

Diana, P. (2013). L'E-Learning in Carcere: Esperienze, Riflessioni e Proposte. Cambio, (6), 261271. https://doi.org/10.1400/218601

Earle, R. (2011). Prison and university: a tale of two institutions? British Society of Criminology, 4-6, 20-37. Extraído el 4 de abril de 2020 desde http://oro.open.ac.uk/30983/1/pbcc_2011_ Earle.pdf

García-Vita, M.M. (2017). ¿Qué sucede con la familia y los vínculos sociales de las mujeres cuando son condenadas a prisión? En F. Añaños-Bedriñana (Dir.), En prisión: realidades e intervención socioeducativa y drogodependencias en mujeres (pp.121-134). Madrid: Narcea.

Gutiérrez Brito, J., Viedma Rojas, A. y Callejo Gallego, J. (2010). Estudios superiores en la educación penitenciaria española: un análisis empírico a partir de los actores. Revista de Educación, 353, 443-468. Extraído el 5 de abril de 2020 desde http://www.educacionyfp.gob.es/dam/jcr:208ee311abbf-4f9d-a1a5-7e70882d663b/re35316-pdf.pdf

Hawley, J., Murphy, I. y Souto-Otero, M. (2013). Prison education and training in Europe. Extraído el 4 de abril de 2020 desde https://www.voced.edu.au/content/ngv\%3A56865

Larrea Álvarez, R. M. (2014). De las políticas penitenciarias invisibilizadas a un nuevo modelo de educación en el sistema carcelario. Fides et Ratio, 8(8), 41-58. Extraído el 4 de abril de 2020 desde http://www.scielo.org.bo/scielo.php?script=sci_arttext\&pid=S2071-081X2014000200004\&lng=e s\&tlng=es.

López, M. (2010). La educación en las cárceles de la provincia de Buenos Aires. Question, 1(27). Extraído el 4 de abril de 2020 desde https://perio.unlp.edu.ar/ojs/index.php/question/article/view/999

Maculan, A., Ronco, D. y Vianello, F. (2013). Prison in Europe: Overview and Trends. Roma: Antigone. Extraídoel 4 de abril de 2020 desde http://www.prisonobservatory.org/upload/ PrisoninEuropeOverviewandtrends.pdf.

Makoe, M. (2016). Analysing Education Policies for the Future: Realising the Vision of Open Distance Learning in 2030. Extraído el 4 de abril de 2020 desde http://oasis.col.org/handle/11599/2595

Moreira, J. A., Reis-Monteiro, A. y Machado, A. (2017). La educación superior a distancia y el e learning en las prisiones en Portugal. Revista Comunicar, 51, 39-49. https://doi.org/10.3916/C51-2017-04

Murillo, W. (2019). La educación universitaria en prisión: logros, dificultades y necesidades. Educación, 43(2). DOI:10.15517/REVEDU.V43I2.31967

ONU (1966). Pacto Internacional de Derechos Económicos, Sociales y Culturales. Extraído el 14 de abril de 2020 desde https://www.ohchr.org/SP/ProfessionalInterest/Pages/CESCR.aspx

Parchuc, J. P. (2015). La Universidad en la cárcel: teoría, debates, acciones. Redes de Extensión, 1, 18-36. Extraído el 4 de abril de 2020 desde https://www.academia.edu/25739424/La_Universidad_en_la_ carcel

Pastore, G. (2018). Inclusion and social exclusion issues in university education in prison: considerations based on the Italian and Spanish experiences. International Journal of Inclusive Education, 22(12), 1272-1287.

Pérez, C. (2019). La Ley Argentina de Estímulo Educativo y la educación en contextos de encierro. Espacios en blanco, 1(30), 147-158. https://doi.org/https://doi.org/10.37177/UNICEN/EB30-269

Rivera, M. (2016). Análisis de los factores que inciden en el rendimiento académico de la población estudiantil privada de libertad de los centros de atención institucional Dr. Gerardo Rodríguez Echeverría, La Leticia y Sandoval. Tesis doctoral no publicada. UNED, Costa Rica. 
Sáez López, J. M., Domínguez Garrido, C. y Mendoza Castillo, V. (2014). Valoración de los obstáculos, ventajas y prácticas del e-learning: un estudio de caso en Universidades Iberoamericanas. Educación Siglo XXI, 32(2), 195-220. https://doi.org/10.6018/j/202221

Scarfó, F. y Zapata, N. (2013). Obstáculos en la realización del derecho a la educación en cárceles. Una aproximación sobre la realidad argentina. Extraído el 4 de abril de 2020 desde http://sedici.unlp.edu.ar/bitstream/handle/10915/69650/Documento_completo.pdfPDFA.pdf?sequence $=1 \&$ isAllowed $=\mathrm{y}$

Schneider, G. (2018). De la educación en cárceles de Argentina y España. Entre el enfoque de derechos y el tratamiento penal. Revista de Estudios Marítimos y Sociales, 12, 10-23. Extraído el 4 de abril de 2020 desde https://estudiosmaritimossociales.org/rems/rems12/ Schneider.pdf

Secretaría General de Instituciones Penitenciarias (2012, 2013, 2014, 2015, 2016, 2017). Informe General. Madrid: Secretaría General de Instituciones Penitenciarias. Extraído el 4 de abril de 2020 desde http://www.institucionpenitenciaria.es/web/portal/documentos/ publicaciones.html

Silva, M. (2020). La dimensión pedagógica de la equidad en educación superior. Archivos Analíticos de Políticas Educativas, 28(46), 1-26. https://doi.org/10.14507/epaa.28.5039

Solé Blanch, J. (2020). El cambio educativo ante la innovación tecnológica, la pedagogía de las competencias y el discurso de la educación emocional. Una mirada crítica. Teoría de la Educación. Revista Interuniversitaria, 32(1), 101-121. doi:10.14201/teri.20945

Sumba, N., Cueva, J. y López, R. (2019). Experiencias en el ejercicio de la educación superior en la prisión, desde la perspectiva del docente. Estudio de caso: Guayaquil, Ecuador. Páginas De Educación, 12(2), 72-88. https://doi.org/10.22235/pe.v12i2.1838

Touriñán López, J.M. (2019). Estudiar es actividad común externa y siempre educamos con la actividad. Una aproximación desde la perspectiva mesoaxiológica. Teoría de la Educación. Revista Interuniversitaria, 31(2), 7-39. doi:10.14201/teri.20571

UNESCO (2008). Educación en prisiones en Latinoamérica: derechos, libertad y ciudadanía. Brasil: UNESCO- OEI-AECID. Extraído el 4 de abril de 2020 desdehttps://www.oei.es/ historico/pdfs/prisiones.pdf

UNESCO (2015). Declaración de Incheon y Marco de Acción para la realización del Objetivo de Desarrollo Sostenible. Garantizar una educación inclusiva y equitativa de calidad y promover oportunidades de aprendizaje permanente para todos. Extraído el 14 de abril de 2020 desde https://unesdoc.unesco.org/ark:/48223/pf0000245656_spa

Vila Merino, E. (2019). Repensar la relación educativa desde la pedagogía de la alteridad. Teoría de la Educación. Revista Interuniversitaria, 31(2), 177-196. doi:10.14201/teri.20271 